\title{
ASPECTS OF LOCALIZED GRAVITY AROUND THE SOFT MINIMA
}

\author{
Pablo M. Llatas 1 \\ Departamento de Física de Partículas \\ Universidad de Santiago \\ Santiago de Compostela, E-15706
}

\begin{abstract}
$n$-Dimensional pure gravity theory can be obtained as the effective theory of an $n+1$ model (with non-compact extra dimension) where general $n+1$ reparametrization invariance is explicitly broken in the extra dimension. As was pointed out in the literature, a necessary consistency condition for having a non-vanishing four dimensional Newton constant is the normalizability in the extra dimension of the zero mass graviton. This, in turn, implies that gravity localization is produced around the local minima of a potential in the extra dimension. We study gravity in the neighborhood of the soft ("thick") local minima.
\end{abstract}

US-FT-01/01

January, 2001

1 llatas@fpaxp1.usc.es 


\section{Introduction}

In [1] (that we will refer to as RSII model) it was shown that five dimensional gravity coupled to a three brane (the "brane-world") produces a geometry confining the zero mass graviton in a small neighborhood of the brane-world, in such a way that the propagation of the massless gravitons are suppressed on the extra dimension. Moreover, the coupling to the brane-world of the continuum spectrum of massive gravitons propagating on the extra dimension was shown to be highly suppressed as well (in the neighborhood of the brane-world). Therefore, the massless gravitons propagate basically just along the braneworld and the massive ones decouple from the brane-world physics. This picture produces a four dimensional effective theory which is the one that we observe (in the spirit of these models) at low energies.

The localization of the massless gravitons and the decoupling of the massive ones were produced by the fact that these gravitons are under the influence of a $\delta$-potential on the extra dimension, a consequence of the special geometry of the model (two copies of AdS-5 pasted along the four dimensional brane-world). The physical interpretation of such a sharp potential becomes from the fact that all the matter was just placed along the four-brane, breaking five-dimensional general invariance in five dimensions with a $\delta(z)$ source.

In [2] the authors studied some properties that general $(n+1)$-dimensional geometries have to fulfill in order to produce confinement of the massless gravitons in the extra dimension, and produce effective theories in one dimension less. One necessary condition was found to be that the massless graviton has to correspond to a normalizable wave on the extra dimension. This corresponds to situations in which the potential to with the gravitons are influenced in the extra dimension has one (or several) minimum. The core of the massless gravitons is placed around the minimum and then, physics can be effectively described as four-dimensional around such minimum, if the massive gravitons decouple.

The RSII picture in which all matter is placed along a four dimensional submanifold of a five dimensional space-time is very suggestive from the string theory point of view, where there exist objects like the $D-3$ branes which can play the role of brane-worlds. This picture lead to metrics which are discontinuous (with a jump on the brane) and, therefore, to singular geometries. However, to obtain localized gravity, the presence of a brane is not a must, and we will study a model where the metric is continuous and smooth at the minima. This restores the derivability condition on the metric and removes the curvature $\delta$-singularity.

In our set up we will have several normalizable graviton modes, with a mass gap between them. In this respect, localized gravity resembles a "string" model. In fact, for the harmonic approximation, we will see that the mass spectrum looks very close to that corresponding to a string with one excitation mode and with tension $\alpha^{\prime} \sim \frac{1}{w}$ (being $w$ the frequency of oscillation). The lighter gravitons in our model should describe a "first order" local approximation to the lighter gravitons of localized gravity with smoothminima potentials. Of course, our massive gravitons will differ substantially from the "true" massive ones at energy scales where the "true" potential sensibly differs from the harmonic one. However, the lightest gravitons are the most sensible to gravitational tests.

We will work out a pure gravity case, in the sense that the dynamical fields in five (and 
four) dimensions are just gravitons. The restriction to five dimensions is not essential in our analysis, but we will take it as the simplest one that produces an effective four-dimensional pure gravity. The model that we will work out is based on the five dimensional action:

$$
S=-\frac{1}{G_{N}^{(5)}} \int d^{4} x d z \sqrt{-G}\left[R+\Lambda(z)+\frac{\mu(z)}{\sqrt{G_{z z}}}\right] .
$$

$\Lambda(z)$ and $\mu(z)$ are source terms on the bulk and the $\mu(z)$ term breaks, explicitly, fivedimensional general reparametrization invariance down to four-dimensional reparametrization invariance along the $z$-direction (the RSII model corresponds to $\Lambda(z)$ constant and $\mu(z)=\lambda \delta(z))$. The action (1.1) can be interpreted as a continuous collection of parallel $D-3$ branes with local tension $\mu(z)$. However, the important point is that the effective four dimensional action derived from this action is just the Einstein-Hilbert action with a cosmological constant term (as we will see bellow).

The organization of the paper is as follows. In section 2 we present the general setting of the models that we are going to study. We comment some general features of localized gravity on soft potentials at high and low four-dimensional energies and also we briefly study experimental bounds to the first massive graviton. In section 3 we analyze localization of gravity by a harmonic confining potential. This is a very interesting model, because it is analytically tractable. We study the decoupling of heavier gravitons due to two facts, first the suppressed coupling of the heavier gravitons to the "thick world-brane"

four-manifold, and second, an exponential suppression of the graviton waves along the extra dimension. We also study the form of the graviton waves in the extra dimension. Finally, in section 4 we present our conclusions and give an outlook of future developments.

\section{General Setting.}

First of all, let us discuss the effective four dimensional theory associated to the action (1.1). For the ansatz:

$$
d s^{2}=e^{2 A(z)}\left(g_{m n}(x) d x^{m} d x^{n}+d z^{2}\right)
$$

(where $z$ denotes the extra dimension) one gets, from (1.1), the equations of motion:

$$
\begin{aligned}
& R_{m n}^{(4)}(x)+3\left(A^{\prime \prime}(z)+A^{\prime}(z)^{2}\right) g_{m n}(x)=\frac{1}{2}\left(e^{2 A(z)} \Lambda(z)+e^{A(z)} \mu(z)\right) g_{m n}(x) \\
& 6 A^{\prime}(z)^{2}-\frac{1}{2} R^{(4)}(x)=\frac{1}{2} e^{2 A(z)} \Lambda(z)
\end{aligned}
$$

(the RSII case [1] is the particular solution $g_{m n}=\eta_{m n}, A(z)=-\operatorname{Ln}(1+k|z|), \Lambda(z)=12 k^{2}$ and $\mu(z)=-12 k \delta(z))$.

Note that the second of these equations imply that $g_{m n}(x)$ must be a metric of constant scalar curvature $R^{(4)}(x)=k$. Therefore, one gets the four dimensional effective action:

$$
S_{e f f}=-\frac{1}{G_{N}^{(4)}} \int d^{4} x \sqrt{-g} R^{(4)}(x)+\Lambda^{(4)} \int d^{4} x \sqrt{-g}
$$


being $R^{(4)}(x)$ the scalar curvature associated to the four dimensional metric $g_{m n}(x)$ and the effective four-dimensional Newton and cosmological constants given by:

$$
\begin{aligned}
& G_{N}^{(4)}=\frac{G_{N}^{(5)}}{\int_{-\infty}^{\infty} d z e^{3 A(z)}} \\
& \Lambda^{4}=-\frac{2}{3 G_{N}^{(5)}} \int_{-\infty}^{\infty} d z \frac{d^{2} e^{3 A(z)}}{d z^{2}}+\frac{k}{2 G_{N}^{(5)}} \int_{-\infty}^{\infty} d z e^{3 A(z)}
\end{aligned}
$$

Demanding the four dimensional effective Newton constant $G_{N}^{(4)}$ to be non-vanishing requires that the function $e^{3 A(z)}$ belongs to the Hilbert space $L^{2}[R]$ ([2]). Then, the boundary contribution to $\Lambda^{(4)}$ in (2.4) vanishes identically. Here, we will study the case where $e^{3 A(z)}$ has just one maximum (which will correspond to the case in which the confining gravitational potential has just one minimum), because we are interested on the local properties of gravity in the neighborhood of a single minimum. Performing a global coordinate translation in the $z$ direction and a global rescaling, we can set this maximum at $z=0$ and fix $A(0)=0$. Then, we get:

$$
\begin{aligned}
G_{N}^{(4)} & =G_{N}^{(5)} \psi_{0}(0)^{2} \\
\Lambda^{(4)} & =\frac{k}{2 G_{N}^{(4)}}
\end{aligned}
$$

where we have defined $\psi_{0}(z)$ as the normalized function of $L^{2}[R]$ :

$$
\psi_{0}(z) \equiv \frac{e^{\frac{3}{2} A(z)}}{\sqrt{\int_{-\infty}^{\infty} d z e^{3 A(z)}}}
$$

In this paper, we concentrate on effective four-dimensional theories where $R^{(4)}(x)=$ 0 , therefore we will take, from now on, $k=0$ and thus, the four dimensional effective cosmological constant identically vanishes.

Let us now study the linear metric perturbations that a gravitational source $j\left(x^{\prime}, z^{\prime}\right)$, in five dimensions, produces around the four-dimensional flat vacuum solution. On the gauge $\gamma_{z z}=\gamma_{n z}=0$ we can take, as the general perturbed metric:

$$
d s^{2}=\left(e^{2 A(z)} \eta_{m n}+\gamma_{m n}(x, z)\right) d x^{m} d x^{n}+e^{2 A(z)} d z^{2}
$$

where $\eta_{n m}$ is the four-dimensional Minkowski metric and we are using the $(-,+,+,+)$ signature convention. Imposing, moreover, the traceless transverse conditions $\gamma_{n}^{n}=\partial_{n} \gamma_{m}^{n}=0$ (where the contractions are performed with $\eta^{n m}$ ) and using standard techniques ([3, 2, , 4]) we arrive at:

$$
\gamma(x, z)=e^{2 A(z)} \int d^{4} x^{\prime} d z^{\prime} \Delta\left(x, z ; x^{\prime}, z^{\prime}\right) j\left(x^{\prime}, z^{\prime}\right)
$$


where we suppressed the Lorentz indices in the previous equation (corresponding to the graviton polarizations) due to the decoupling of the equations on the chosen gauge. $\Delta\left(x, z ; x^{\prime}, z^{\prime}\right)$ is the retarded propagator $\left(t>t^{\prime}\right)$ given by:

$$
\Delta\left(x, z ; x^{\prime}, z^{\prime}\right)=e^{-\frac{3}{2}\left(A(z)-A\left(z^{\prime}\right)\right)} \sum_{n=0}^{\infty} \psi_{n}(z) \psi_{n}\left(z^{\prime}\right) T_{n}\left(x, x^{\prime}\right)
$$

where $\psi_{n}(z)$ are the normalized eigenfunctions of the operator

$$
\mathcal{O}_{z}[A] \equiv \frac{d^{2}}{d z^{2}}-\frac{3}{2} A^{\prime \prime}(z)-\left(\frac{3}{2}\right)^{2} A^{\prime}(z)^{2}
$$

with eigenvalues $-m_{n}^{2}$ :

$$
\mathcal{O}_{z}[A] \psi_{n}(z)=-m_{n}^{2} \psi_{n}(z)
$$

(in such a way that the sum in (2.9) has to be understood as an integration on the continuum part of the spectrum) and $T_{n}\left(x, x^{\prime}\right)$ is the standard four dimensional scalar retarded propagator for a particle of mass $m_{n}$ :

$$
T_{n}\left(x, x^{\prime}\right)=-\frac{\theta\left(t-t^{\prime}\right)}{2 \pi}\left(\delta\left[-\left(x-x^{\prime}\right)^{2}\right]-\frac{m_{n}}{2} \frac{\theta\left[-\left(x-x^{\prime}\right)^{2}\right]}{\sqrt{-\left(x-x^{\prime}\right)^{2}}} J_{1}\left[m_{n} \sqrt{-\left(x-x^{\prime}\right)^{2}}\right]\right) .
$$

(Here, $\theta$ is the step function $\theta(x)=0$ for $x \leq 0$ and $\theta(x)=1$ for $x>0,-\left(x-x^{\prime}\right)^{2}=$ $\left(t-t^{\prime}\right)^{2}-\left(\bar{x}-\bar{x}^{\prime}\right)^{2}$ and $J_{1}(x)$ is the first Bessel function). The first term in $T_{n}\left(x, x^{\prime}\right)$ is the massless contribution with support on the four-dimensional light-cone and the second one is the contribution of the massive modes with support inside the light-cone.

$\mathcal{O}_{z}[A]$ is an Hermitean operator that can be written as ([2])

$$
\mathcal{O}_{z}[A]=-W_{z}[A] W_{z}^{\dagger}[A]
$$

being

$$
W_{z}[A]=-\frac{d}{d z}-\frac{3}{2} A^{\prime}(z) .
$$

Therefore, one gets that the spectrum of $\mathcal{O}_{z}[A]$ is negative definite, in such a way that $m_{n}^{2} \geq 0$ in (2.11). Also, the normalized massless zero mode is the normalized solution of the equation $W_{z}^{\dagger}[A] f(z)=0$, which can be easily integrated to give (2.6).

One can compute the static retarded propagator $\Delta\left(\bar{x}, z ; \bar{x}^{\prime}, z^{\prime}\right)$ from equations $(2.9)$ and $(2.12)$. The result is:

$$
\Delta\left(\bar{x}, z ; \bar{x}^{\prime}, z^{\prime}\right) \equiv \int_{-\infty}^{\infty} d t^{\prime} \Delta\left(x, z ; x^{\prime}, z^{\prime}\right)=-\frac{e^{-\frac{3}{2}\left(A(z)-A\left(z^{\prime}\right)\right)}}{4 \pi r} \sum_{n=0}^{\infty} e^{-m_{n} r} \psi_{n}(z) \psi_{n}\left(z^{\prime}\right) .
$$

(here, $r=\left|\bar{x}-\bar{x}^{\prime}\right|$ ), where we get the standard Yukawa couplings. This lead to the static metric perturbation (produced by a static source)

$$
\gamma(\bar{x}, z)=-\frac{1}{4 \pi} e^{\frac{1}{2} A(z)} \int_{-\infty}^{\infty} d \bar{x}^{\prime} d z^{\prime} \frac{e^{\frac{3}{2} A\left(z^{\prime}\right)}}{r} \sum_{n=0}^{\infty} e^{-m_{n} r} \psi_{n}(z) \psi_{n}\left(z^{\prime}\right) j\left(\bar{x}^{\prime}, z^{\prime}\right) .
$$


From the previous expression it is clear that, at low energy experiments (large $r$ ), the lighter gravitons are the relevant ones. Also we see the physical relevance of the four dimensional submanifold $z=0$ (where we have set the maximum of the function $e^{A(z)}$ ). First, the $j\left(\bar{x}^{\prime}, z^{\prime}\right)$ part with support off the $z=0$ plane is suppressed by the factor $e^{\frac{3}{2} A\left(z^{\prime}\right)}$. Second, the propagation of gravitational perturbations are suppressed off that plane by a factor $e^{\frac{1}{2} A(z)}$ as well. In this sense one says that gravity is localized around the $z=0$ plane and, by abuse of language, we call this plane a "thick world-brane".

Note also that the linear approximation will remain valid even if we approach the high energy four-dimensional limit ( $r$ small) for strong enough decreasing functions $e^{A(z)}$. The closer we go to the $r \rightarrow 0$ limit, the farther we have to go in $z$ to maintain the linear approximation valid $(z \rightarrow \infty)$. In other words, the fifth dimension becomes more apparent as we perform higher four dimensional experiments maintaining the graviton (i.e., linear) approximation in our description of the physical processes.

Let us compute now the post-Newtonian law that an observer in five dimensions perceives. Introducing the local perturbation $j\left(\bar{x}^{\prime}, z^{\prime}\right)=G_{N}^{(5)} M \delta^{(3)}\left(\bar{x}^{\prime}\right) \delta\left(z^{\prime}\right)$ on equation (2.16) one gets:

$$
\gamma(\bar{x}, z)=-\frac{G_{N}^{(5)} M}{4 \pi r} e^{\frac{A(z)}{2}} \sum_{n=0}^{\infty} e^{-m_{n} r} \psi_{n}(z) \psi_{n}(0) .
$$

(where now $r=|\bar{x}|$ ). In particular, for a four dimensional observer living in the $z=0$ four-manifold:

$$
\gamma(\bar{x}, 0)=-\frac{G_{N}^{(4)} M}{4 \pi r}\left(1+\frac{1}{\psi_{0}(0)^{2}} \sum_{n=1}^{\infty} e^{-m_{n} r} \psi_{n}(0)^{2}\right) .
$$

(where we have used (2.5)).

The previous equation resembles a "mass renormalization" (of a four dimensional Quantum Field Theory)2, being the "bare" mass $M$ and the "renormalized" (radius dependent) mass $M^{*}(A, r)$ defined through:

$$
M^{*}(A, r)=M\left(1+\frac{1}{\psi_{0}(0)^{2}} \sum_{n=1}^{\infty} e^{-m_{n} r} \psi_{n}(0)^{2}\right) .
$$

(the dependence of $M^{*}$ on $A$ is due to the dependence on the spectrum of the operator $\mathcal{O}_{z}[A]$ in $\left.(2.10)\right)$. Note that, very far from the source, $\lim _{r \rightarrow \infty} M^{*}(A, r) \rightarrow M$, as the only gravitons that can reach such distances are the massless ones. This result does not depend on the function $A(z)$, which means that the details of the geometry in the fifth dimension is becoming fuzzy to asymptotic four-dimensional observers. On the other hand, on the ultrashort distances $\lim _{r \rightarrow 0} M^{*}(A, r) \rightarrow \frac{1}{\psi_{0}(0)^{2}} M \delta(0)$ (where we have used the formal identity $\left.\sum_{1}^{\infty} \psi_{n}(0)^{2} "=" \delta(0)-\psi_{0}(0)^{2}\right)$. Therefore, the mass corrections are becoming stronger at shorter distances for observers on the "world brane". Note, however, that the linear approximation to $\gamma(\bar{x}, 0)$ becomes unjustified at distances of the order of the "dressed horizon" $G_{N}^{(4)} M^{*}\left(A, r_{0}\right) \sim r_{0}$, as $\gamma(\bar{x}, 0)$ in (2.18) is not small. (In fact, the linear

2 We find a formal analogy with the renormalized Coulomb law in four dimensional QED. 
approximation to the pure post-Newtonian law (discarding the massive modes) becomes also unfair at distances shorter that the order of the "bare horizon" $\left.r \sim G_{N}^{(4)} M\right)$.

We already commented above that linear approximations at ultrashort distances can be only justified if $A(z)$ is strong enough and always at large $z$ distances (i.e., in five dimensions). Now we can make this statement more precise. Let us go beyond the "horizon" (i.e., to the $r<G_{N}^{(4)} M$ region) maintaining the linear approximation valid (i.e., in five-dimensional space). From (2.17) we have that

$$
\frac{G_{N}^{(4)} M}{r} e^{\frac{A(z)}{2}} \sim 1 \Rightarrow A(z) \sim 2 \operatorname{Ln}\left(\frac{r}{G_{N}^{(4)} M}\right)
$$

therefore, the linear approximation is valid if $A(z)$ is smaller than $\sim 2 \operatorname{Ln}\left(\frac{r}{G_{N}^{(4)} M}\right)$. Being $e^{A(z)}$ normalizable, this means big $z$.

Let us say a word on experimental bounds to the Yukawa-like corrections to the post-Newtonian law. From (2.18) it is clear that the first Yukawa correction to the postNewtonian law is given by the first massive graviton (which we call $\psi_{*}(z)$ ) which couples to the manifold $z=0$ (i.e., such that $\psi_{*}(0) \neq 0$ ). Moreover, this Yukawa correction is certainly the most important one if the higher mass modes couple less to $z=0$ than $\psi_{*}(z)$ (i.e., if $\psi_{*}(0)^{2}>\psi_{n}(0)^{2} \forall n>*$ ). This must be the case for localized gravity.

In reference [5], experimental bounds for Yukawa-like corrections to the gravity potential of the form:

$$
V(r)=-\alpha N_{1} N_{2} \hbar c \frac{e^{-\frac{r}{\lambda}}}{r}
$$

are studied for a Casimir-force experiment. $\alpha$ is a dimensionless parameter, $N_{1}$ and $N_{2}$ are the numbers of nucleons in the atomic nuclei which are interacting gravitationally in the experimental setting, and $\lambda$ is a parameter with dimensions of length (in meter units). The experimental bound is a curve on the $(\alpha, \lambda)$-plane. The regions of the $(\alpha, \lambda)$-plane below that curve are permitted, and above are ruled out by experiments (see figure 3 of [5]).

In our present case, we have to compare 2.21) with the first Yukawa correction that the post-Newtonian law (2.18) gives for the gravitational interaction between two nuclei with $N_{1}$ and $N_{2}$ nucleons:

$$
v(r)=-\frac{G_{N}^{(4)} m_{N}^{2} N_{1} N_{2}}{4 \pi r} \frac{\psi_{*}(0)^{2}}{\psi_{0}(0)^{2}} e^{-m_{*} r}
$$

(note that the corrections of the higher mass modes are smaller due both to the exponential mass suppression and the $z=0$ coupling suppression). For our case, then, $\alpha$ and $\lambda$ are given by:

$$
\alpha \sim 5 \cdot 10^{-40} \frac{\psi_{*}(0)^{2}}{\psi_{0}(0)^{2}}, \quad \lambda=\frac{1}{m_{*}} .
$$

Because we are assuming that gravity is localized, $\frac{\psi_{*}(0)^{2}}{\psi_{0}(0)^{2}}<1$, then we have that $\alpha<$ $5 \cdot 10^{-40}$. Therefore this shows that this scenario can not be tested by the done pure gravity 
experiments (Casimir, Cavendish and Eotvos-type, which reach values over $\alpha \sim 10^{-39}$ ) in [5].

On the other hand, very recently, a different experiment has been studied in [6] which is able to set a lower bound on $m_{*}$. In their notation, the Yukawa correction to the gravitational potential is parametrized by

$$
V(r)=-G_{N}^{(4)} \frac{m_{1} m_{2}}{r}\left(1+\alpha e^{-\frac{r}{\lambda}}\right)
$$

In our case, and from figure 4 of [6], we then get:

$$
\alpha=\left(\frac{\psi_{*}(0)}{\psi_{0}(0)}\right)^{2}<1 \Rightarrow \lambda_{*}<2,5 \cdot 10^{-4} \text { met. } \Rightarrow m_{*}>7.5 \cdot 10^{-4} \mathrm{eV} .
$$

Note that pure gravitational test puts a very low lower bound on the mass of the lightest graviton that couples to the "thick world-brane".

\section{The Harmonic Oscillator Approximation.}

Demanding that the zero mode of the spectrum associated to the linear operator $\mathcal{O}_{z}[A]$ in $(2.10)$ is normalizable, translates to the fact that the "Schrodinger"-like potential in equation (2.11) has (at least) a minimum. The extreme case in which the only normalizable mode is the massless mode corresponds to the RSII model, where the $\delta(z)$-potential can locate just one bound state. Certainly, from a conventional General Relativity point of view, it is a more pleasant picture to have a potential that is not that sharp, and that it is smooth also at the minimum. This lead us to trade (see also [7,2]) the $\delta(z)$ potential by a smooth and derivable potential. This, in turn, maintains (apart of continuity) a derivability property on the five-dimensional geometry (in such a way that we do not have to worry about Israel matching conditions as in the AdS cases). At this point, we can approximate (locally) the gravitational confining potential around its minima in the $z$ direction by the harmonic oscillator potential. The energies up to which this approximation is faithful depend on the energy scale at which the "true" potential differs significantly from the harmonic one. But at least, locally, the massless graviton should be accurately described by the ground state of the harmonic oscillator (and perhaps, some of the first massive gravitons). Remember that, precisely, the smaller the masses of the gravitons are, the longer they can travel to large "world-brane" distances (see (2.18), where we measure the deviations to the Newtonian law. Therefore, the light gravitons are the most relevant ones for experimental purposes.

Let us mention at this point that the exact spectral resolution of the problem (2.11) is known for some few potentials. In particular, we have the "Shape Invariant Potentials" of supersymmetric quantum mechanics [8] (one of those is the harmonic case). But we do not expect them to change significantly the local picture (at least for the light gravitons) around the soft minima of the potential.

Therefore, let us take

$$
A(z)=-\frac{w}{3} z^{2}
$$


in equation (2.11). The spectral resolution to this equation is given by:

$$
\psi_{n}(z)=\left(\frac{w}{\pi}\right)^{\frac{1}{4}} \frac{1}{\sqrt{2^{n} n !}} e^{-\frac{w}{2} z^{2}} H_{n}(\sqrt{w} z), \quad m_{n}=\sqrt{2 n w},
$$

where $H_{n}(x)$ are Hermite polynomials. A few comments are in order. First of all, from equations (2.5) and (3.2), the harmonic frequency of vibration can be expressed in terms of the ratio of the Newton constants $G_{N}^{(4)}$ and $G_{N}^{(5)}$ as:

$$
\frac{G_{N}^{(4)}}{G_{N}^{(5)}}=\sqrt{\frac{w}{\pi}} .
$$

Second, the mass spectrum (3.2) is very similar to the mass spectrum of a string with one excitation mode and tension

$$
m_{s t r}^{2} \sim \frac{n}{\alpha^{\prime}} \Rightarrow \alpha^{\prime} \sim \frac{1}{w} .
$$

In fact, the graviton wave-modes along the extra dimension can be interpreted as the vibrating modes of a "string", and the mass associated to a given mode is proportional to its number of oscillation. A difference with string-theory is that, there, the strings are of finite length and one imposes Newmann or Dirichlet boundary conditions. In our present case, the wave satisfy the ("finite energy") boundary conditions on the extra dimension (see (2.8) and (2.9)) $\lim _{z \rightarrow \pm \infty} \gamma(x, z)=0$ which are analogous to a string configuration with two D3-branes at $z \rightarrow \pm \infty$ with a string attached to them.

Also, from (3.2), the mass spectrum of the gravitons tends to be closer as one increases the energy $m_{n+1}-m_{n} \sim \sqrt{\frac{w}{2 n}}$. Finally, the odd modes of the gravitons $(n$ odd) do not couple to the four dimensional brane-world at $z=0$ (just because $H_{2 n+1}(0)=0$ ). Therefore, the highest correction to the Newton law comes from the $n=2$ graviton.

Let us then discuss the experimental bound to the frequency of vibration of the harmonic oscillator (i.e., to the ratio of the four dimensional effective Newton constant and the five-dimensional Newton constant). Because, for the harmonic case, $\alpha=1 / 2$ (in the notation of [6]), the mass bound for the lightest massive graviton does not change substantially the result in (2.25). In particular, one gets $m_{2}>5 \cdot 10^{-4} \mathrm{eV}, w>6 \cdot 10^{-8} \mathrm{eV}^{2}$ and $G_{N}^{(4)}>1.4 \cdot 10^{-4} G_{N}^{(5)} \mathrm{eV}$. Note that this mass bound is very small compared with the electron mass!, and yet, it is compatible with done pure gravity experiments.

In spite of that we are working with an uncompactified extra dimension, from (2.5) one can define $R \equiv \psi(0)^{-2}$ as the analog of "Kaluza-Klein compactification radius". Then, from (3.2) and the experimental lower bound for $w$ that we got, we obtain an experimental upper bound for $R$ :

$$
R<9 \cdot 10^{-4} \mathrm{~m} \sim 1 \mathrm{~mm}
$$

which is concordant with the results in the literature in which the pure gravity experiments are compatible with extra dimensions with a compactification scale lower than $1 \mathrm{~mm}$.

Note that a "unification" five-dimensional Newton constant (with a five-dimensional Planck mass of the order of a $\mathrm{TeV}$ ) is deep inside the experimentally ruled-out region, 
because $\frac{G_{N}^{(4)}}{G_{N}^{(5) G U T}} \sim 10^{-21} \mathrm{eV}$. This is concordant also with the unification models of [9], which require two or more extra dimensions.

Let us now discuss the contribution of the massive modes to the post-Newtonian law. From equation (3.2) we get that the odd modes decouple completely from $z=0$, and the coupling of the even modes to $z=0$ are driven by (see (2.19))

$$
c_{2 n}(r) \equiv e^{-m_{2 n} r}\left(\frac{\psi_{2 n}(0)}{\psi_{0}(0)}\right)^{2} \equiv e^{-m_{2 n} r} \lambda_{2 n} .
$$

Using the doubling formula for the Euler Gamma function, we arrive at:

$$
\lambda_{2 n}=\frac{1}{\sqrt{\pi}} \frac{\Gamma\left(n+\frac{1}{2}\right)}{\Gamma(n+1)} .
$$

It is clear that there are two suppressing factors for the contribution to the post-Newtonian law when the graviton mass increases. The first one $\left(\lambda_{2 n}\right)$ is due to the fact that the coupling (to the "world-brane" at $z=0$ ) of the eigenfunctions of the spectral problem (2.11) decreases as we increase $n$. The second one is due to the exponential suppression with the "world-brane" distance $r$ along the $z=0$ plane $e^{-m_{2 n} r}$. This two suppression factors is what we have drawn in figure 1, for the first lighter fifty gravitons: $\lambda_{2 n}$ (in gray) and $c_{2 n}(r)$ for $r=1 \mathrm{~mm}$ (in black), and for the most sensitive value of $w$ compatible with the experimental data in [6] $\left(2 \sqrt{w} \sim 10^{3} \mathrm{~m}^{-1}\right)$. At $r=1 \mathrm{~cm} c_{2 n}(r)$ is a number smaller than $10^{-8}$ for the gravitons with $n \geq 1$, therefore, at these energies only the massless graviton couples to "brane-world" physics.

Due to the discrete character of the graviton spectrum, we do not face problems with the van Dam-Veltman-Zakharov discontinuity ([10,11, 12, 13]). In our case the contribution of the first massive-mode to the bending of light by the sun has a suppression factor $e^{-2 \sqrt{w} r}$, that, for the lower bound on $w\left(2 \sqrt{w} \sim 10^{3} \mathrm{~m}^{-1}\right)$ and distances bigger than the sun's radius, is completely negligible.

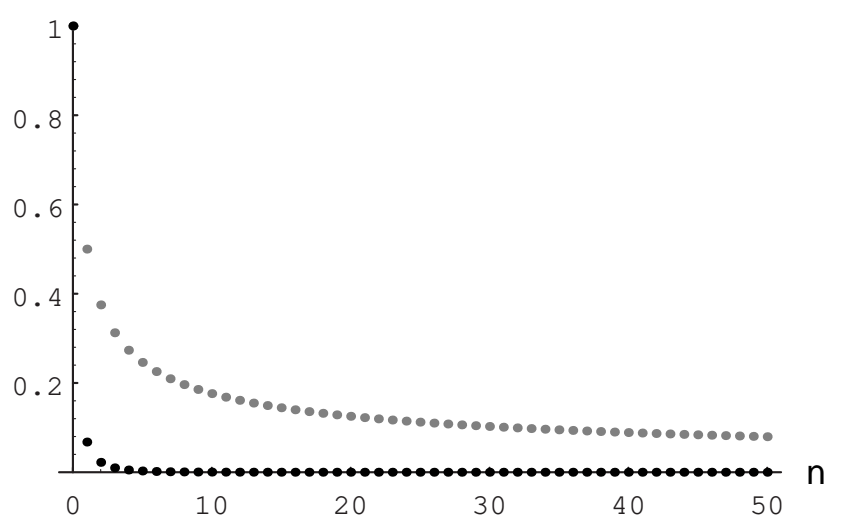

Fig. 1: In gray, $\lambda_{2 n}$ for the first fifty gravitons. In black, $c_{2 n}(r)$ for $r=1 \mathrm{~mm}$ and $2 \sqrt{w}=10^{3} \mathrm{~m}^{-1}$. For $r=1 \mathrm{~cm}, c_{2 n}(r)$ is negligible for $n \geq 1$. 
Let us now analyze the form of the graviton waves. In equation (2.17) we observe that the $z$ dependence of the graviton modes corresponds to that of the eigenfunctions of (2.11) suppressed by an exponential factor $e^{\frac{A(z)}{2}}$. Moreover, the dependence with the distance $r$ from the source along the $z=0$ plane is given by the Yukawa factors times $\frac{1}{r}$. From equations (2.17), (3.1) and (3.2) we get:

$$
\mu_{2 n} \equiv \frac{1}{r} e^{\frac{A(z)}{2}} \sum_{n=0}^{\infty} e^{-m_{n} r} \psi_{n}(z) \psi_{n}(0)=(-)^{n} \frac{e^{-2 \sqrt{n w} r}}{2^{2 n} \Gamma(n+1) r} e^{-\frac{2}{3} w z^{2}} H_{2 n}(\sqrt{w} z)
$$

In figure 2 we draw $\mu_{2 n}$ and compare the graviton waves (for $r=1 \mathrm{~mm}$ and $r=1 \mathrm{~cm}$ and the same mass source $M$ ) for the first three modes that couples to the four-manifold at $z=0$. We have taken, again, the experimental lower bound for $w\left(2 \sqrt{w} \sim 10^{3} \mathrm{~m}^{-1}\right)$. Also, we have drawn in gray the corresponding harmonic oscillator quantum wave-function (normalized such that its value on $z=0$ coincides with the $r=1 \mathrm{~mm}$ graviton wave), in such a way that we have an explicit picture of the $e^{\frac{A(z)}{2}}$ suppression factor in (2.17). In this figure, the gravitons $2 n=2,4$ at $1 \mathrm{~cm}$ do not appear because their amplitudes are $10^{-8}$ and $10^{-12}$ (respectively) smaller than for the case of $1 \mathrm{~mm}$.
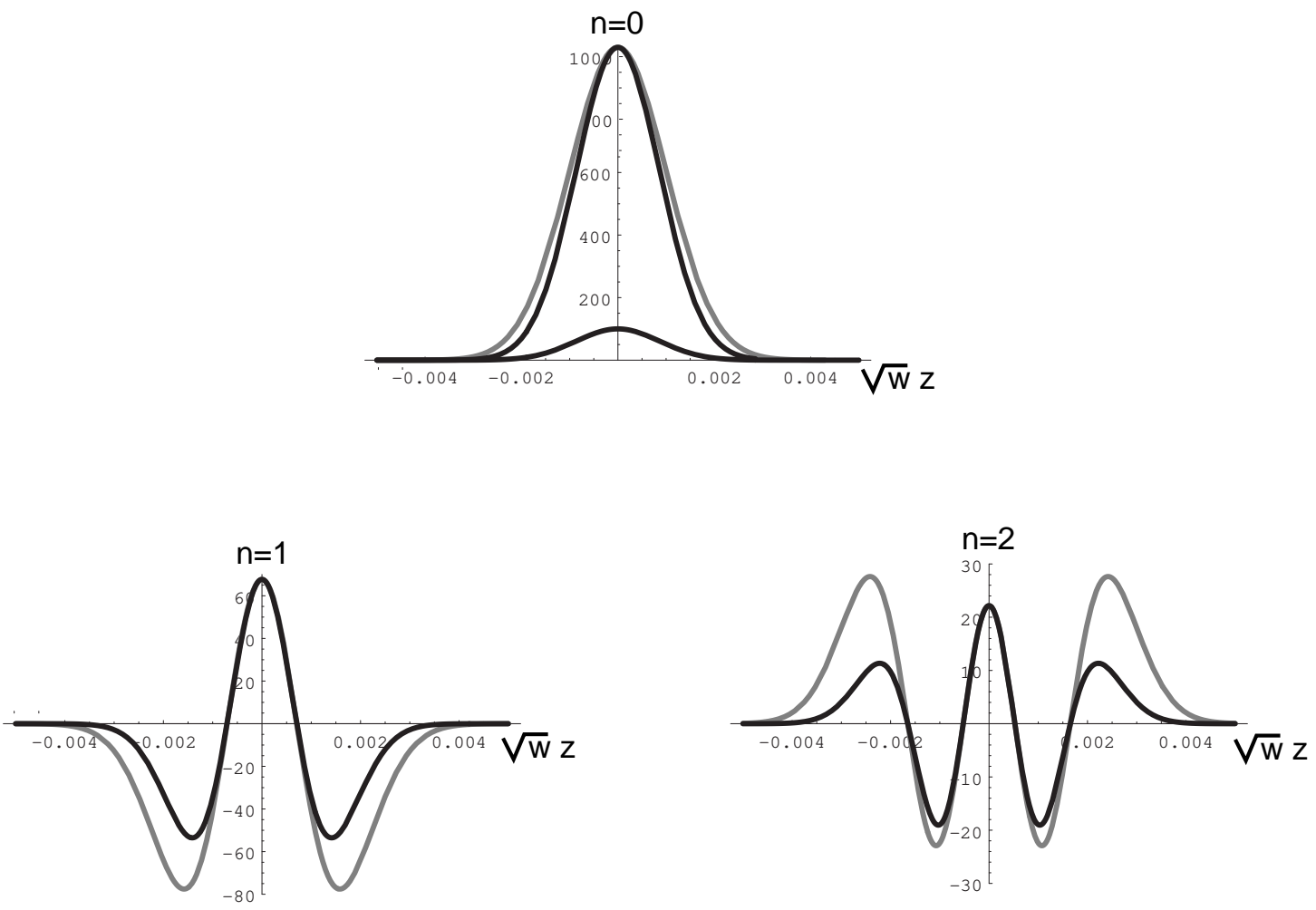

Fig. 2: In gray, quantum harmonic oscillator wave-functions (properly normalized). In black, $\mu_{2 n}(n=0,1,2)$ for $r=1 \mathrm{~mm}$ (the highest ones) and $r=1 \mathrm{~cm}$ (the lower ones).

For $n=1,2$ the amplitudes of the gravitons at $r=1 \mathrm{~cm}$ are negligible. 
We can also naturally introduce a notion of the "width" (at "brane-world" distance $r$ from the source) along the $z$ direction of the graviton waves $\gamma_{2 n}(\bar{x}, z)$ (the ones that couple to four dimensional physics) through $\left.l_{2 n}(r) \equiv\left(<z^{2}\right\rangle_{2 n}\right)^{\frac{1}{3}}$ being

$$
<z^{2}>_{2 n} \equiv \int_{-\infty}^{\infty} d z z^{2} \gamma_{2 n}(\bar{x}, z)^{2} .
$$

Substituting equations (2.17) and (3.2) in (3.9) we arrive at:

$$
\begin{aligned}
<z^{2}>_{2 n} & =\frac{1}{w^{\frac{3}{2}}}\left(\frac{G_{N}^{(4)} M e^{-2 \sqrt{n w} r}}{4 \pi r} \frac{1}{2^{2 n} \Gamma(n+1)}\right)^{2} \Gamma\left(2 n+\frac{1}{2}\right)\left(\frac{3}{4}\right)^{\frac{5}{2}} \times \\
& {\left[\left(\frac{2}{3}-8 n\right) F\left[-2 n,-2 n ; \frac{1}{2}-2 n ; 2\right]-\frac{64 n^{2}}{4 n-1} F\left[1-2 n, 1-2 n ; \frac{3}{2}-2 n ; 2\right]\right] }
\end{aligned}
$$

being $F[\alpha, \beta ; \gamma ; \delta]$ Gauss's Hypergeometric series. As an example, from (3.10) we can obtain the ratio of the lengths corresponding to the massless graviton and the first two massive ones which couples to the "world-brane", obtaining:

$$
\frac{<z^{2}>_{0}}{<z^{2}>_{2}}=\frac{64}{79} e^{4 \sqrt{w} r} \quad \frac{<z^{2}>_{0}}{<z^{2}>_{4}}=\frac{16384}{12753} e^{4 \sqrt{2 w} r}
$$

We see that, for large $r$, the thickest graviton on the extra dimension is the massless one .

In figure 3 we draw the $n$-dependent part of $<z^{2}>_{2 n}$ in de range $0 \leq n \leq 15$, for $r=1 \mathrm{~mm}$ and $2 \sqrt{w} \sim 10^{3} \mathrm{~m}^{-1}$. Again, at $r=1 \mathrm{~cm}$, the size of the gravitons with $n \geq 1$ is negligible for this $w$.

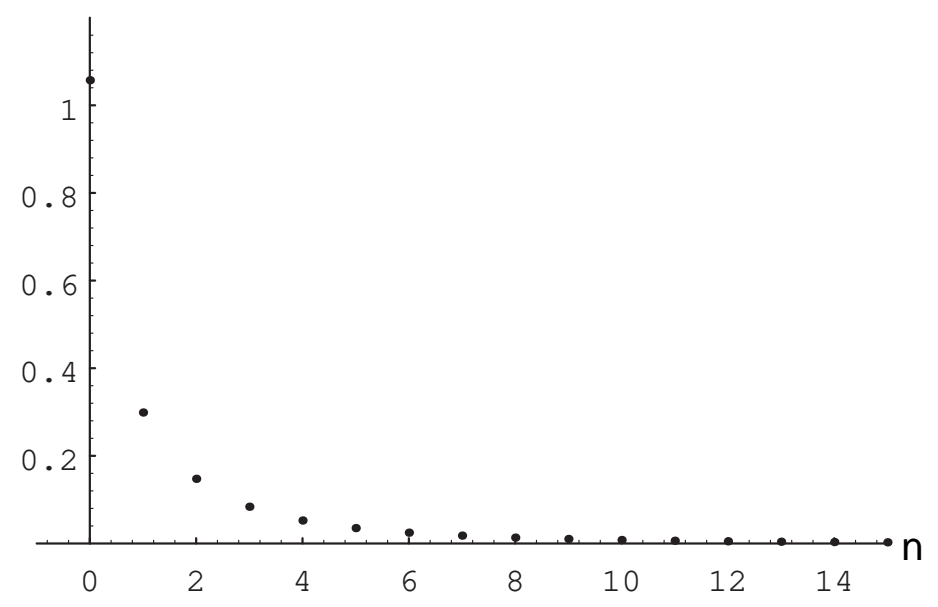

Fig. 3: n-dependent part of $<z^{2}>_{2 n}$ for the first fifteen lighter gravitons.

\section{Conclusions and Outlook.}

We have studied general aspects of localized gravity of pure gravity "thick-branes". In the literature, it has been suggested that the RSII-type models can be observationally best tested in pure gravity experiments. We have obtained an experimental lower 
bound to the mass of the graviton which produces the biggest contribution to deviations to the Newtonian law. We have studied the local harmonic approximation to pure gravity localization (for four-dimensional geometries of zero scalar curvature) and obtained the expression (3.3) for the frequency of oscillation in terms of the ratio of the four-dimensional effective Newton constant and the five-dimensional Newton constant. As we have argued, the harmonic approximation for the zero mode (and maybe, some of the lighter ones) is a "first-order" description of the "exact" first graviton modes for "thick-branes" (those that can allocate several normalizable modes), in the neighborhood of the minima of the confining gravitational potential. We have studied for this approximation (and analytically) the increasing relative decoupling of the massive modes to four dimensional physics and the form of the graviton waves. We have also introduced a notion of size for the graviton waves on the extra dimension and found its analytical expression. The heavier, the smaller they are in the extra dimension (due to the two exponential suppression factors in (3.8)).

There are several issues that remain for further research. One of them is to study "localized thermodynamics" by taking a graviton thermal-bath configuration as a source in (2.16) instead of a $\delta(z)$ source (profiting of the fact that the harmonic case is analytically tractable). This means to impose that the physics on the extra dimension is a quantum one, in contrast with the classical one that we have studied $\left(\delta(z)=\sum_{n=0}^{\infty} \psi_{n}(z) \psi_{n}(0)\right.$ are not density matrix entries). The extra dimension model could provide us some hints on black-hole thermodynamics and information problem.

Also, it would be interesting to introduce dynamics on the problem. This can be done introducing time-dependent sources in (2.8), studying time-dependent background metrics $(A(z) \rightarrow A(z, t))$ or studying transition amplitudes between the graviton states by using perturbation theory up to order $\gamma(x, z)^{2}$.

Another question is the implications of the picture presented here for cosmology and the cosmological constant. We have seen that, for the models described by the action (1.1), the cosmological constant identically vanishes for four-geometries of vanishing scalar curvature (it is a surface term). In the past years, experimental evidence for non-vanishing four-dimensional cosmological constant has emerged. Therefore, the study of the cases in which the four-geometry in (2.1) is of constant (but non-vanishing) scalar curvature (being the four-dimensional effective cosmological constant in (2.5) not vanishing) is phenomenologically relevant. Moreover, the localized models that we have explored contain massive modes for the gravitons, whose effects on four dimensional physics are highly suppressed already at decimeter scales (by experimental bounds). Therefore, the Newtonian law does not change at astronomic scales, but we could have cosmological implications (as this massive gravitons mimic "dark matter"). A cosmological analysis could provide an experimental upper bound for $m_{*}$ and refine the lower bound that we have found.

\section{Acknowledgments}

We would like to thank Roberto Emparan, Jose M. Sánchez de Santos and Ricardo Vázquez for valuable comments and for a critical reading of the manuscript. This work was supported in part by DGICYT under grant PB96-0960 and by the Xunta de Galicia grant PGIDT00-PXI-20609. 


\section{References}

[1] L. Randall and R. Sundrum, Phys.Rev.Lett. 83 (1999) 4690-4693, hep-th/9906064.

[2] C. Csaki, J. Erlich, T.J. Hollowood and Y. Shirman, Nucl.Phys. B581 (2000) 309-338, hep-th/0001033.

[3] J. Garriga and T. Tanaka, Phys.Rev.Lett. 84 (2000) 2778-2781, hep-th/9911055.

[4] S.B. Giddings, E.Katz and L. Randall, JHEP 0003 (2000) 023, hep-th/0002091.

[5] M.Bordag, B.Geyer, G.L. Klimchitskaya and V.M. Mostepanenko, Phys.Rev. D58 (1998) 075003, hep-ph/9804223.

[6] C.D. Hoyle, U. Schmidt, B.R. Heckel, J.H. Gundlach, D.J. Kapner and H.E. Swanson, hep-ph/0011014.

[7] M. Gremm, Phys.Lett. B478 (2000) 434-438, hep-th/9912060.

[8] F. Cooper, A. Khare and U. Sukhatme, Phys. Rep. 251 (1995) 267-385..

[9] N. Arkani-Hamed, S. Dimopoulos and G. Dvali, Phys.Rev. D59 (1999) 086004, hep$\mathrm{ph} / 9807344$.

[10] H. van Damm and M. Veltman, Nuc. Phys. B22 (1970) 397; V. I. Zakharov, JETP Lett. 12 (1970) 312.

[11] A. Higuchi, Nucl. Phys. B282 (1987) 397; B325 (1989) 745.

[12] M. Porrati, hep-th/0011152.

[13] I.I. Kogan, S. Mouslopoulos and A. Papazoglou, hep-th/0011138. 